\title{
CARGA DE TRABAJO AUTÓNOMO ESTIMADA POR EL ESTUDIANTE DE MAGISTERIO EN ASIGNATURAS DE CIENCIAS. EXPLORACIÓN DE SU RELACIÓN CON EL RENDIMIENTO ACADÉMICO
}

\author{
Marta Ceballos Aranda, José Eduardo Vílchez López y \\ Teresa Escobar Benavides
}

\section{RESUMEN}

Se presenta un análisis de las discrepancias existentes en la valoración del trabajo autónomo en asignaturas de ciencias, entre estudiantes y profesorado de magisterio. Para ello se ha estimado la carga de trabajo dedicada por el estudiante, así como la que este consideraría óptima en actividades pertenecientes a guías de trabajo, y se han comparado con la planificación del profesorado. Los resultados indican que el estudiante otorga menos importancia a actividades diseñadas para generar nuevo conocimiento, $y$ en cambio, considera de mayor importancia actividades de perfil tradicional como ejercicios de aplicación, trabajo con apuntes o preparación de exámenes. Además una mayor consideración de estas actividades se relaciona con un menor rendimiento académico.

Palabras clave: Carga de trabajo del estudiante, formación inicial de maestros, ECTS, guías de trabajo, innovación docente universitaria.

\section{TITLE: AUTONOMOUS WORKLOAD ESTIMATED BY TEACHER TRAINEES IN SCIENCE SUBJECTS. EXPLORATION OF ITS RELATION WITH ACADEMIC PERFORMANCE}

\begin{abstract}
We present an analysis of existing discrepancies between students and teacher trainers in valuing autonomous work in science subjects. To do this we have estimated the workload carried out by students, as well as what they would consider the most appropriate workload for activities in students' workbooks. We have compared these with teachers' planning. The results indicate that the students give less importance to activities which are designed to generate new knowledge, and on the other hand they consider traditional activities such as application exercises, work with class notes or examination preparation to be more important. Furthemore, a greater consideration of these activities correlates with lower academic performance.
\end{abstract}

Keywords: Students' Workload, Initial Teacher Training, ECTS, Workbooks, University Teaching Innovation.

Marta Ceballos Aranda <mceballos@ceuandalucia.es>.José Eduardo Vílchez López<jvilchez@ceuandalucia.es>. Teresa Escobar Benavides < tescobar@ceuandalucia.es>. Centro de Estudios Universitarios Cardenal Spínola CEU (adscrito a Universidad de Sevilla). Original recibido: 16-0I-I5. Original aceptado: 22-07-I5. 


\section{Introducción y marco teórico}

El desarrollo y adaptación de recursos y metodologías docentes que contemplen la planificación del trabajo autónomo del estudiante universitario ha adquirido una especial importancia estos últimos años, durante el proceso de implementación de las titulaciones derivadas del Espacio Europeo de Educación Superior (EEES). En nuestro grupo de investigación también hemos realizado este tipo de trabajo (Ceballos, Escobar y Vílchez, 20I0; Escobar, Vílchez y Ceballos, 20I2; Vílchez y Escobar, 2009). Para ello consultamos bibliografía básica relacionada con el EEES (De Miguel, 2005; Goñi, 2005; Villa y Poblete, 2007), así como experiencias recientes de innovación en el ámbito de la enseñanza de las ciencias (Andrade, 2007; Melendro y col., 2008, entre otras). También nos familiarizamos con ciertos aspectos del diseño instruccional (Guàrdia y Sangrà, 2005) y tuvimos en cuenta información obtenida a partir de otras líneas de investigación en la que estudiamos las clases reales de ciencias en Educación Primaria (Vílchez y Escobar, 20I4).

No obstante, nos preocupa el impacto real de estos procesos de innovación docente sobre el estudiante. De hecho es conocida la resistencia a cambios metodológicos innovadores por parte de estudiantes y profesores en el ámbito de la enseñanza de las ciencias (Furió y Vilches, 1997; Banet, 2007).

En este contexto juega un importante papel la estimación de la carga de trabajo real del estudiante universitario (student workload), entendida esta como el tiempo total que emplea en actividades presenciales (clases, seminarios, tutorías, entrevistas, exámenes) y trabajo autónomo, incluyendo, proyectos, estudio, preparación de evaluaciones, etc. (Kember, 2004).

Recientemente este concepto ha tenido en nuestro entorno una importante revitalización relacionada con la adaptación de planes de estudio al EEES y al sistema de créditos ECTS (European Credit Transfer System). Recordemos que el sistema ECTS inició su implantación en España en 2003 (Real Decreto, I I 25/2003) como unidad de transferencia de créditos, pero adquirió verdadera importancia con el desarrollo de las nuevas titulaciones universitarias (grados y posgrados) y el proceso de Convergencia Europea (European Commission, 2005; Goñi, 2005). Cada curso universitario se estructuró en 60 ECTS, de forma que I ECTS resultara equivalente a 25-30 horas de trabajo del estudiante. Se inició entonces en las universidades españolas un importante trabajo para asignar la dedicación del estudiante a las distintas tareas, especialmente en lo concerniente a distribuir las presenciales junto al profesor y las no presenciales. Finalmente, en cuanto a las 
titulaciones de Grado se adquirió el consenso de valorar los momentos presenciales en torno a un $30-40 \%$ del total.

Todo este esfuerzo ha generado un importante volumen de resultados que puede encontrarse en la literatura científica y sobre innovación docente universitaria correspondientes a la última década. En ocasiones, la estimación de la carga de trabajo del estudiante aparece integrada junto con otros recursos en un estudio general en el que se aborda la evaluación de la innovación desde distintos puntos de vista (Laborda y Martínez, 2007; Plo y col., 2006). En cambio, con frecuencia aparece como protagonista de la investigación (Esteve y col., 2009; García y col., 2006; Laborda y Martínez, 2006; Martínez y col., 2006; Rožman, 20I4; Ruiz-Gallardo, Valdés y Castaño, 2006; Sánchez y col., 2008; Stepišnik y col., 2007). Normalmente se trata de estudios en los que se intenta valorar, con mayor o menor detalle, la distribución de la carga docente del estudiante a lo largo de un periodo de tiempo. Esta información se utiliza para determinar la relación entre la carga de las diferentes tareas antes de diseñar nuevas asignaturas, para comprobar su similitud con lo previamente planificado y poder así realizar ajustes, o para mejorar la coordinación entre el profesorado de diferentes grupos.

En otros trabajos, de mayor interés para nuestro propósito, se enfatiza más claramente en relacionar la estimación de la carga de trabajo con aspectos metodológicos, el ambiente de aprendizaje, el éxito de los estudiantes o la calidad educativa (Chambers, 1992; Kember, 2004; Kember y col., 1996; Pogacnik y col., 2004; Ruiz-Gallardo y col., 20I I). Nosotros también pensamos que esta técnica puede ser útil más allá del contexto actual de implantación de los nuevos títulos y del sistema ECTS, y constituir un indicador de la adecuación de la metodología planificada por el docente respecto al trabajo real del estudiante y el impacto en su rendimiento académico. Nuestro estudio se ha centrado en el trabajo autónomo del estudiante, excluyéndose el trabajo realizado por este en presencia del profesor. De esta forma, la comparación entre la distribución del esfuerzo que realiza el estudiante fuera de los momentos presenciales, y de cómo éste concibe su trabajo respecto a lo diseñado por el profesor, indicaría finalmente el impacto real de determinados aspectos, especialmente los relacionados con innovación educativa, que el docente puede concebir como ventajosos y explícitos en su planificación. 


\section{Objetivos y metodología}

El estudio se ha desarrollado en el Centro de Estudios Universitarios Cardenal Spínola CEU (centro adscrito a la Universidad de Sevilla) y ha implicado a estudiantes de la Diplomatura de Magisterio (plan de estudios extinguido) y del Grado de Educación Primaria. En este trabajo se presentan datos correspondientes a los primeros años de implantación del nuevo Grado cursos 2010 a 2012 procedentes del análisis de 340 cuestionarios que valoran, cada uno de ellos, el tiempo relacionado con una guía de trabajo.

Los objetivos planteados han sido los siguientes:

- Estimar la carga de trabajo autónomo del estudiante así como la que éste considera óptima para cada uno de los tipos de actividades considerados.

- Analizar las discrepancias encontradas entre las cargas discentes reales y la planificación realizada por los docentes.

- Explorar las posibles relaciones entre estas diferencias y el rendimiento académico del estudiante.

Se dispone de una herramienta para el registro de los datos, en la que se han volcado las siguientes variables para cada tipo de actividad:

- Tiempo dedicado por el estudiante (TD).

- Tiempo considerado óptimo por parte del estudiante (TO).

- Tiempo planificado por el profesorado (TP).

Obviamente en los dos primeros casos los datos son introducidos por el estudiante de acuerdo a su propia estimación, mientras que el tercero es cumplimentado por el profesorado según lo indicado en las guías de trabajo y demás instrumentos de planificación. Para la cuantificación de la planificación del trabajo autónomo se han tenido en cuenta los siguientes criterios seguidos en el Centro:

- I ECTS equivale a 25 horas de trabajo total del estudiante.

- El $40 \%$ se planifican como presenciales: I ECTS implica 10 horas presenciales.

- Las 15 horas restantes se consideran trabajo autónomo del estudiante.

- Todas las actividades planificadas se consideran como obligatorias. 
- En todas las guías de trabajo consideradas (incluidas las de asignaturas de la Diplomatura) la planificación se ha hecho siguiendo este sistema ECTS.

Las asignaturas y guías de trabajo implicadas en la investigación han sido las siguientes:

Curso 2010/II: Ciencias de la Naturaleza y su Didáctica (9 créditos; Guías de trabajo: Didáctica, Física y Química), Educación Medioambiental (6 créditos) y Alimentación y Nutrición (4,5 créditos).

Curso 2011/12: Ciencias de la Materia (6 créditos; Guías de trabajo: Física y Química).

Tal como se ha mencionado anteriormente, el interés fundamental de este trabajo es utilizar la información aportada por las estimaciones de carga docente para conocer el impacto de la planificación metodológica establecida por el profesorado. Por tanto no nos ha interesado profundizar en las diferencias entre Diplomatura y Grado, o entre las diferentes asignaturas y asignaciones de créditos. Para independizar la investigación de estos aspectos se han tomado dos decisiones:

- En primer lugar utilizar como unidad de estudio las Actividades incluidas en las Guías de Trabajo. Estas estaban presentes tanto en Diplomatura como en Grado, así como en diferentes asignaturas. Se han estructurado a efectos de este estudio en las siguientes 7 categorías:

Búsqueda y tratamiento de la información, Informes de laboratorio, Ejercicios de respuesta abierta, Ejercicios de respuesta cerrada, Resolución de problemas, Preparación de exámenes y entrevistas y Trabajo con apuntes relacionados con clases magistrales.

- En segundo lugar, se han tenido en cuenta tiempos relativos en lugar de absolutos (como se verá más adelante), para independizar los resultados del número de actividades y de la duración de las mismas.

El registro de datos se ha completado tras la finalización de cada una de las guías de trabajo de las asignaturas. 


\section{Resultados y discusión}

3.I. Estimaciones de la carga docente y comparación con el estudio piloto

A partir de las hojas de registro cumplimentadas por los estudiantes, se tabulan los tiempos absolutos (horas) para cada tipo de actividad en las tres variables consideradas (Tiempo dedicado: TD, Tiempo óptimo: TO y Tiempo planificado: TP). No obstante, dado que no todos los tipos de actividades aparecen en todas las guías de trabajo y que el volumen de créditos asociados a éstas también es distinto, se ha optado, para independizar las variables de estos aspectos, por la consideración de tiempos relativos (dividiendo en cada caso los tiempos registrados por el estudiante entre el tiempo planificado por el profesor). Tenemos así las variables relativas Tiempo dedicado respecto al planificado (Td) y Tiempo óptimo respecto al planificado (To):

$$
T d=\frac{T D}{T P} ; T o=\frac{T O}{T P} ; T p=\frac{T P}{T P}=1
$$

En la Tabla I se incluyen las medias correspondientes a estas variables relativas. Los valores de estas variables son respectivamente las horas dedicadas o consideradas óptimas por cada hora planificada por el profesor. El valor I indica que el tiempo dedicado u óptimo coincide con el planificado.

\begin{tabular}{r|c|c|c}
\hline Tipo de actividad & $\mathbf{N}$ & Media Td & Media To \\
\hline búsqueda y tratamiento de Información & 290 & 0,63 & 0,83 \\
\hline informes de laboratorio & 244 & 0,91 & 1,09 \\
\hline ejercicios de respuesta abierta & 143 & 0,58 & 0,78 \\
\hline ejercicios de respuesta cerrada & 50 & 1,06 & 1,52 \\
\hline resolución de problemas & 244 & 0,95 & 1,24 \\
\hline preparación de exámenes & 340 & 1,01 & 1,36 \\
\hline trabajo con apuntes & 227 & 1,25 & 1,64 \\
\hline
\end{tabular}

Tabla I. Medias de las variables tiempo dedicado y tiempo óptimo por hora de tiempo planificado

A partir de ahora emplearemos estas variables relativas, por lo que cualquier referencia a "tiempos dedicados" o "tiempos óptimos" deberá entenderse no en términos absolutos sino en relación a lo planificado por el profesor. 
En la Figura I se realiza una comparación entre estos datos y los correspondientes a un estudio exploratorio preliminar (Ceballos, Escobar y Vílchez, 20I0).

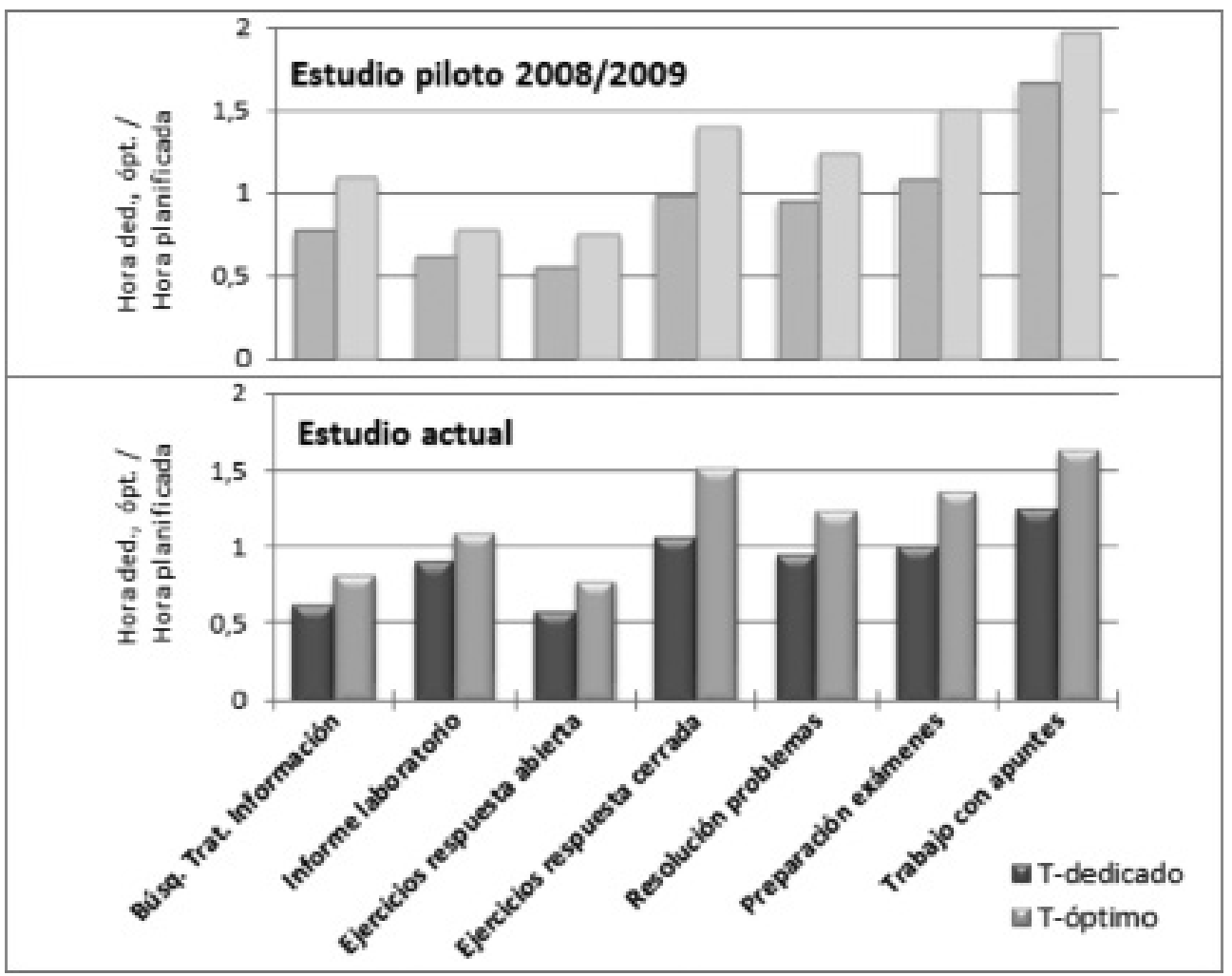

Figura I. Comparación de resultados entre el estudio actual y la experiencia piloto

Como puede comprobarse, el patrón de resultados de ambos estudios es bastante similar, lo que apunta a un comportamiento coherente del procedimiento de análisis. Este hecho nos genera, además, una mayor confianza a la hora de interpretar los resultados. La principal diferencia entre las dos series de datos afecta al tipo de actividad informes de laboratorio, que en la muestra actual recibe mayor consideración por parte de los estudiantes. Esto es lógico ya que la asignatura del Grado de Educación Primaria Ciencias de la materia (no existente en el estudio piloto) incorpora una mayor carga relativa de actividades de laboratorio. 
3.2. Comparación entre los tiempos dedicados, óptimos y planificados. Análisis de las discrepancias

Para visualizar las discrepancias existentes entre las variables, se han considerado para cada tipo de actividad las diferencias entre las medias registradas por los estudiantes y las planificadas:

DifTd, Tp $=\boldsymbol{T d}-\boldsymbol{T p}=\frac{T D-T P}{T P}=\boldsymbol{T d}-1 ; \quad$ DifTd,To $=$ To $-\boldsymbol{T p} \boldsymbol{p}=\frac{T O-T P}{T P}=\boldsymbol{T o}-1$

Asívalores positivos indican tiempos dedicados u óptimos superiores a los planificados y valores negativos indican tiempos dedicados u óptimos inferiores a lo planificado (ver Figura 2). En este caso el valor cero indica coincidencia con el tiempo planificado.

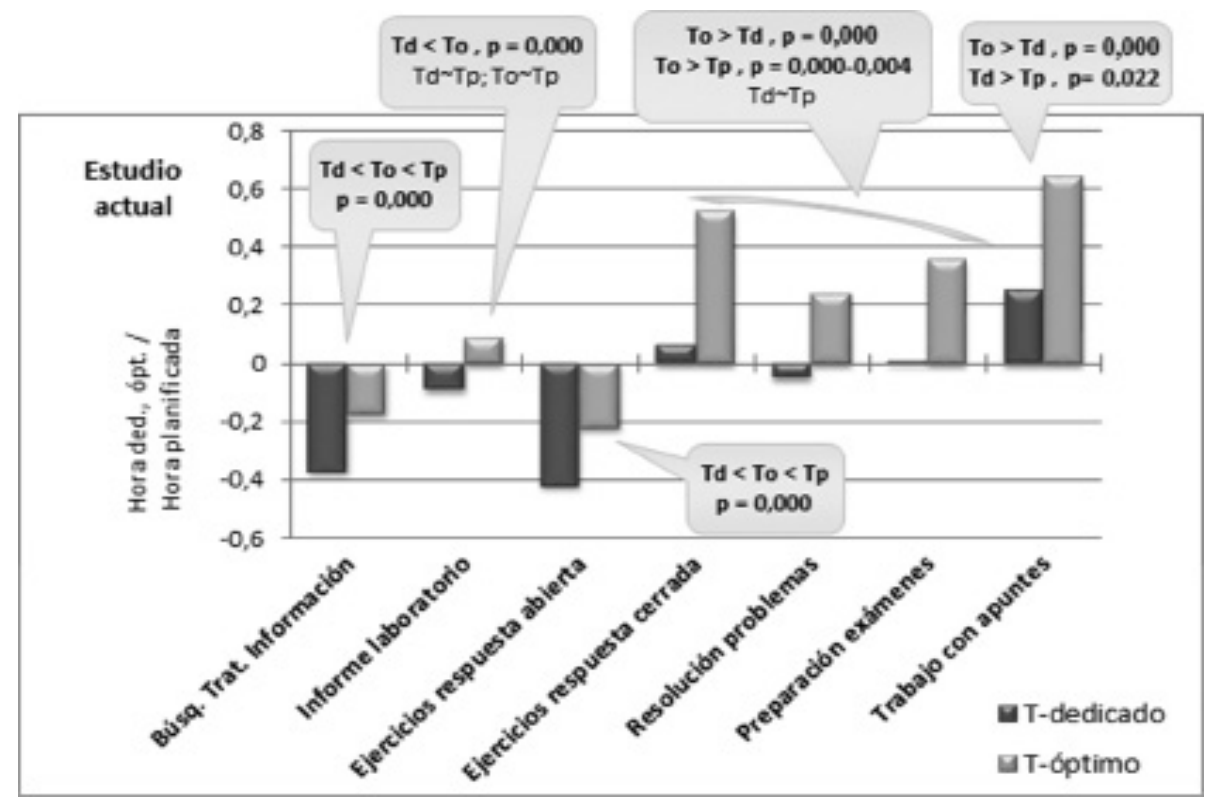

Figura 2. Diferencias entre las medias de los tiempos dedicado y óptimo respecto al planificado.

Además, se ha estudiado la significatividad estadística de estas diferencias comparando entre sí las medias de las variables Td, To y Tp para cada tipo de actividad. Para ello se ha utilizado la prueba de contraste $T$ de Student para muestras relacionadas. En la Figura 2, se indica la significación encontrada en cada caso (los resultados completos de la prueba se resumen en el Anexo I). 
En general puede comprobarse cómo en todos los tipos de actividades considerados el tiempo que el estudiante considera óptimo ha resultado superior al que realmente ha dedicado.

En actividades destinadas a generación de conocimiento como las incluidas en las categorías búsqueda y tratamiento de información y ejercicios de respuesta abierta el tiempo dedicado por el estudiante ha sido significativamente inferior al que considera óptimo y ambos, a su vez, significativamente inferiores al planificado por el profesorado ( $p=0,000$ en todos los casos).

En la categoría informes de laboratorio, si bien el tiempo considerado óptimo resulta (como en todos los tipos de actividades) superior al dedicado por los estudiantes $(p=0,000)$, cada uno de ellos no difiere sustancialmente del planificado por el profesorado ( $p>0,05$ en ambos casos; ver Anexo I).

Otro tipo de actividades de perfil más tradicional siguen una tendencia bastante distinta. Así las categorías ejercicios de respuesta cerrada, resolución de problemas y preparación de exámenes presentan un patrón común consistente en que el tiempo dedicado alcanza al planificado por el profesorado $(p>0,05$; ver Anexo I), pero ambos son significativamente inferiores al que los estudiantes consideran óptimo ( $\mathrm{P}$ $=0,000-0,004$ ). En el tipo de actividad trabajo con apuntes también se detectan estas diferencias, pero además en este caso el tiempo dedicado por los estudiantes supera significativamente al planificado $(p=0,022)$.

3.3. Exploración de la relación entre el rendimiento académico de los estudiantes y el ajuste de su carga docente a la planificada por el profesorado

Para abordar este nuevo objetivo, se ha explorado si existe relación entre las diferencias de los tiempos dedicados o considerados óptimos respecto a los planificados (variables independientes), y las calificaciones que los estudiantes obtienen en cada actividad (variable dependiente).

Para ello se han realizado las correspondientes correlaciones bivariadas, empleando el coeficiente de correlación de Pearson. Se han considerado las diferencias tanto en su valor absoluto como en su valor real (positivo o negativo). Mediante las primeras, se valorará si el ajuste a la planificación del profesorado se asocia con un mejor rendimiento académico del estudiante. Mediante las segundas veremos el efecto que tiene el aumento de la carga docente dedicada u óptima (desde las inferiores a las planificadas hasta las superiores a las mismas). 
Se ha considerado cada tipo de actividad en cada guía de trabajo para cada estudiante. Se han tenido en cuenta las calificaciones disponibles sobre las pruebas de evaluación a las que contribuían dichas actividades. El estudio puede considerarse exploratorio, puesto que aunque las pruebas consideradas son las más directamente relacionadas con cada tipo de actividad, no se dispone de la contribución concreta de cada actividad a esa calificación.

En la Tabla 2 se resumen los resultados de estas correlaciones bivariadas, tanto en su versión general, como desglosados para cada una de las 7 categorías de actividades (los resultados detallados se muestran en el Anexo 2). Las 4 columnas ( $A, B, C$ y D) corresponden a los 4 tipos de correlaciones consideradas:

A: Calificación. Actividad vs $\mid$ DifTd,Tp | / B: Calificación. Actividad vs $\mid$ DifTo,Tp

C: Calificación. Actividad vs DifTd,Tp / D: Calificación. Actividad vs DifTo,Tp

Se indica si existe correlación o no, su nivel de significación, así como el sentido de la misma. Positiva si un aumento en las diferencias supone un aumento en la calificación, y negativa si un aumento en las diferencias supone una disminución en la calificación.

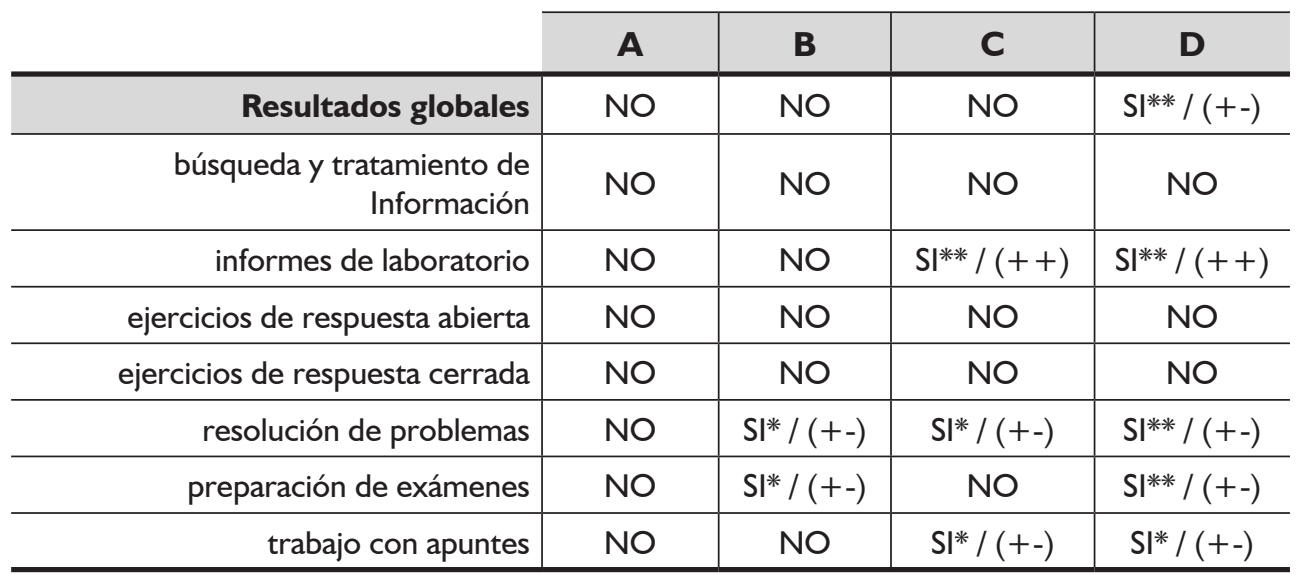

A: Calificación. Actividad vs $\mid$ DifTd,Tp | B: Calificación. Actividad vs $\mid$ DifTo,Tp|

C :Calificación. Actividad vs DifTd,Tp / D: Calificación. Actividad vs DifTo,Tp

NO: No existe correlación estadísticamente significativa

SI*: Correlación significativa a nivel $\alpha=0,05$ / SI*** Correlación significativa a nivel $\alpha=0,0$ I

$(++)$ : Correlación positiva / (+ -): Correlación negativa

Tabla 2. Resumen de las correlaciones bivariadas entre las calificaciones y las diferencias de los tiempos dedicados y óptimos con los planificados. 
Los resultados globales (incluyendo todos los tipos de actividades) indican que no existe correlación cuando se emplean las diferencias en valor absoluto. Tampoco cuando se utiliza la diferencias entre los tiempos dedicados y planificados. En cambio sí existe correlación negativa, con alta significatividad estadística ( $\alpha$ $=0,0 \mathrm{I}$ ) cuando se emplean las diferencias entre tiempos óptimos y planificados (columna D). Es decir, cuanto mayor es el tiempo que el estudiante considera que debería haber dedicado de forma óptima menores son las calificaciones obtenidas y viceversa.

Esta misma tendencia se repite, cuando contemplamos las correlaciones según las distintas categorías de actividades en el caso de resolución de problemas, preparación de exámenes (a nivel $\alpha=0,0 \mathrm{I})$ y trabajo con apuntes $(\alpha=0,05)$, todas ellas de perfil tradicional. En el caso de resolución de problemas y trabajo con apuntes, ese tipo de correlación también se observa cuando se consideran la diferencia entre tiempo dedicado y tiempo planificado (columna C). Por tanto en estas categorías de actividades un mayor tiempo dedicado o considerado óptimo por el estudiante se traduce en peores calificaciones.

El análisis de las correlaciones a partir de las diferencias en valor absoluto (columnas A y B) ha sido menos productivo. Sólo en el caso de las categorías resolución de problemas y preparación de exámenes se han encontrado correlaciones, también negativas (con nivel de significación $\alpha=0,05$ ). En estos tipos de actividades un mayor ajuste entre el tiempo que el estudiante considera óptimo y el planificado por el profesorado supone un mayor rendimiento del estudiante y viceversa.

En el caso de los informes de laboratorio, las correlaciones encontradas son de tendencia contraria (positivas) a las descritas anteriormente. Los estudiantes que señalan mayores tiempos dedicados u óptimos alcanzan mayores calificaciones y viceversa. Esto puede deberse a la singularidad en la evaluación de esta actividad en la que el componente grupal es muy importante, de forma que el tiempo de trabajo autónomo individual tiene menos impacto que en el resto. De todas formas, también nos hace plantearnos al profesorado sobre la pertinencia en la modalidad de evaluación y/o en nuestra planificación de la carga de trabajo para esta categoría.

En la Figura 3 se muestran algunos ejemplos gráficos de dispersión de puntos y ajuste para algunas de las correlaciones estudiadas que han resultado significativas: 
- I: Calificación vs diferencias de tiempo óptimo (Dif_To,Tp) en los resultados globales

- II: Calificación vs diferencias de tiempo óptimo (Dif_To,Tp) en la categoría preparación de exámenes.

- III: Calificación vs diferencias de tiempo óptimo (Dif_To,Tp) en la categoría resolución de problemas.

- IV: Calificación vs diferencias de tiempo dedicado (Dif_Td,Tp) en la categoría informes de laboratorio.

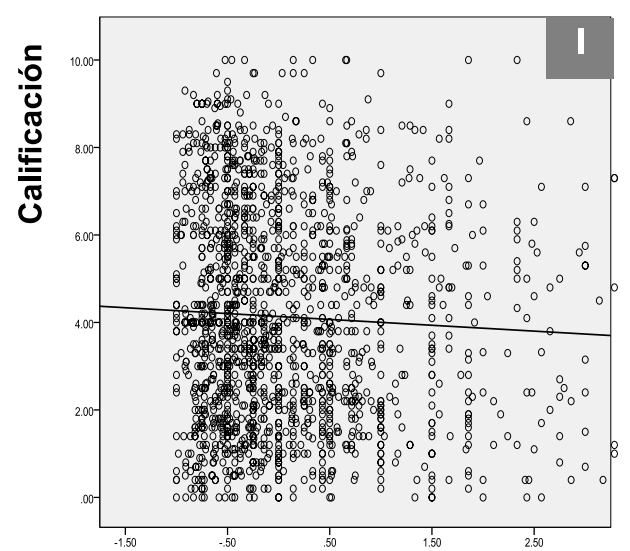

Dif_To,Tp (total)

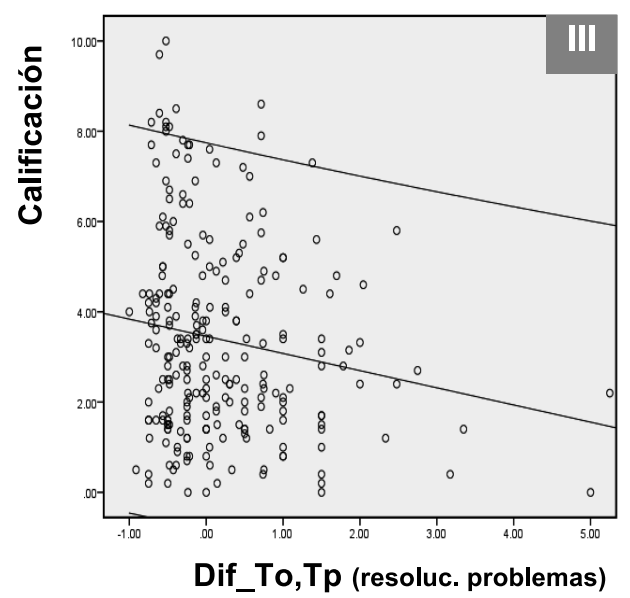

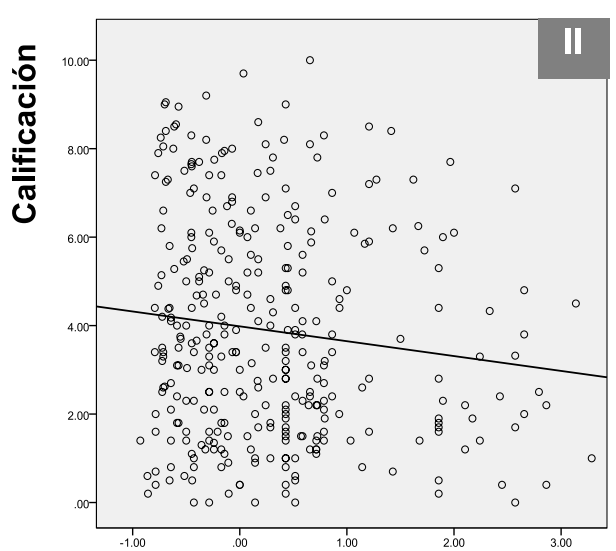

Dif_To,Tp (prep. exámenes)

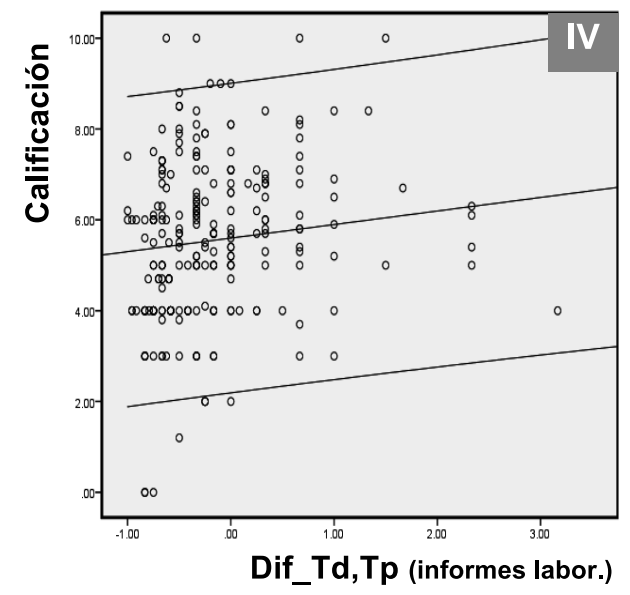

Figura 3. Ejemplos de gráficos de dispersión de puntos y ajuste. 


\section{Conclusiones y reflexiones finales}

Se ha reunido información sobre el tiempo autónomo empleado por estudiantes de magisterio en diversos tipos de actividades incluidas en guías de trabajo de asignaturas de ciencias. Se ha distinguido entre el tiempo que el estudiante admite haber dedicado realmente y el que consideraría óptimo. De esta forma se tiene información sobre la importancia que el estudiante otorga a cada tarea independientemente del tiempo que le haya podido dedicar. Se ha comparado esta carga de trabajo autónomo que el estudiante de magisterio dedica o consideraría óptimo dedicar con el planificado por el profesorado.

Se ha encontrado que el estudiante otorga menos importancia de la planificada por el profesor a actividades para generar nuevo conocimiento (gestión de información, ejercicios de respuesta abierta) y en cambio considera que debe emplear más tiempo de su trabajo autónomo en actividades de tipo más tradicional como ejercicios de aplicación, trabajo con apuntes y preparación de evaluación. El primer grupo de actividades son las que, por su diseño, están más orientadas a responder a las necesidades de regulación del trabajo autónomo del estudiante acorde con el EEES y el sistema ECTS, contribuyendo a desarrollar competencias básicas relacionadas con la gestión del propio aprendizaje. El segundo grupo de actividades incluye tareas también importantes, aunque su sobrevaloración por parte de los estudiantes puede indicar una concepción del conocimiento como "algo terminado" que debe ser aportado por el profesor y posteriormente "practicado o aprendido" por el estudiante. De hecho esta alta consideración por enfocar el trabajo autónomo como preparación de la evaluación, se ha detectado en grupos que siguen metodologías tradicionales, especialmente al final del periodo de clases (Ruiz-Gallardo y col., 20 I I).

En definitiva, aunque el estudiante pueda reconocer los progresos de metodologías de innovación y responder positivamente ante recursos novedosos, se resiste a dejar de considerar su trabajo autónomo de acuerdo a un perfil clásico del proceso de enseñanza-aprendizaje.

Se ha explorado si estas discrepancias entre lo considerado importante por parte del estudiante y la planificación docente afecta al rendimiento académico. En el caso de actividades destinadas a generar conocimiento no se han encontrado correlaciones, pero sí en el caso de algunas de las actividades de corte más tradicional. Así, las calificaciones obtenidas disminuyen en estudiantes que tienden a dedicar más tiempo a resolución de problemas numéricos y gestión de apuntes 
- que considerarían óptimo emplear más tiempo en este tipo de actividades y en la preparación de pruebas de evaluación. Podemos calificar a este tipo de estudiante como "poco realista" no siendo capaz de valorar adecuadamente la carga de trabajo necesaria para mejorar su rendimiento académico, considerando a veces como necesarios tiempos exageradamente altos. En cambio estudiantes que alcanzan superiores calificaciones ajustan mejor su carga de trabajo en este tipo de actividades.

Las tareas relacionadas con la elaboración de informes de laboratorio ofrecen unos resultados singulares. Por una parte es la única categoría de actividades en la que tanto los tiempos dedicados como los considerados óptimos no difieren significativamente de los planificados. También es la única en la que existe una correlación positiva de forma que mayores tiempos dedicados u óptimos implican una mejor calificación. Puede que el hecho de que esta actividad sea fundamentalmente grupal distorsione la percepción del trabajo individual o bien que necesite una reconsideración de la carga planificada.

En su conjunto, las relaciones encontradas entre la carga de trabajo y el rendimiento académico están lejos de responder a un patrón simplista consistente en un aumento en las calificaciones al ampliarse la carga de trabajo autónomo como quizás ingenuamente podría esperarse. En este sentido los resultados son coherentes con otros estudios que han intentado valorar esta relación desde otros puntos de vista (Pogacnik, 2004).

Parece conveniente en cualquier caso, una mejor metacognición del proceso de aprendizaje por parte del estudiante, al menos en lo que respecta a lo que el profesorado persigue en su planificación. Es importante enfatizar esto al presentar las guías de trabajo autónomo, de forma que aspectos que el docente concibe como ventajosos e innovadores queden claros también para los estudiantes.

\section{Referencias bibliográficas}

Andrade, A. (2007). Aprendizaje combinado como propuesta en la convergencia europea para la enseñanza de las ciencias naturales. E-Learning Papers, 3. Recuperado el 0I/05/2015 de <http://www.researchgate.net/profile/Antonia Andrade/publication/28I50025_Aprendizaje_combinado_como_propuesta en_la_convergencia_europea_para_la_enseanza_de_las_ciencias_naturales/ links/0fcfd5 I $017 \mathrm{~d} 45499 \mathrm{ca000000.pdf>}$ 
Banet, E. (2007). Finalidades de la educación científica en secundaria: opinión del profesorado sobre la situación actual. Enseñanza de las Ciencias, 25 (I), 2-20, y referencias allí citadas.

Ceballos, M., Escobar, T. y Vílchez, J. E. (2010). Estimación de la carga de trabajo autónomo del estudiante de magisterio en asignaturas de ciencias. En A. M. Abril y A. Quesada (Eds.), Actas de los XXIV Encuentros de Didáctica de las Ciencias Experimentales (pp. 86-91). Baeza: Universidad de Jaen.

Chambers, E. (1992). Workload and the quality of student learning. Studies in Higher Education, 17 (2), I4I-I53.

De Miguel, M. (Dir.) (2005). Modalidades de enseñanza centradas en el desarrollo de competencias. Orientaciones para promover el cambio metodológico en el EEES. Madrid: MEC/Universidad de Oviedo.

Escobar, T., Vílchez, J. E. y Ceballos, M. (20I2). Influencia del ajuste de la carga de trabajo autónomo del estudiante de magisterio sobre su rendimiento académico. En J. M. Domínguez (Ed.), XXV Encuentro de Didáctica de las Ciencias Experimentales (pp. 293-300). Santiago de Compostela: Universidad de Santiago de Compostela.

Esteve, J. M., Molina, M. A. y Stephens, J. (2009). A qualitative assessment of students' experiences of studying music: a Spanish perspective on the European credit transfer system (ECTS). Music Education Research, I I (2), 24I-265.

European Commission (2005). ECTS Users's guide. European credit transfer and accumulation system and the diploma supplement. European Commission. Directorate General for Education and Culture. Recuperado el 0I/05/20I5 de <http://www.unze.ba/download/ECTSguide_en.pdf>.

Furió, C. y Vilches, A. (1997). Las actitudes del alumnado hacia las ciencias y las relaciones ciencia, tecnología sociedad. En L. Del Carmen, (Coord.), La enseñanza y el aprendizaje de las ciencias de la naturaleza en la educación secundaria. Barcelona: Horsori.

García, P., Hernández, A., Martínez, J. P., Martínez, I., Mayordomo, E., Ortega, A., Salinas, I., Solera, J. y Vicente, L. (2006). Estudio sobre la carga de trabajo del estudiante en las titulaciones del Centro Politécnico Superior. En I Jornadas de Innovación Docente, Tecnologías de la Información y la Comunicación e Investigación Educativa en la Universidad de Zaragoza. Zaragoza, España. Recuperado el 0I/05/20 I5 de <http://diec.unizar.es/ imr/personal/docs/Indoteclnfo06.pdf >. 
Goñi, J. M. (2005). El espacio europeo de educación superior, un reto para la universidad. Barcelona: Octaedro.

Guàrdia L. y Sangrà (2005). Diseño instruccional y objetos de aprendizaje; hacia un modelo para el diseño de actividades de evaluación del aprendizaje on-line. RED. Revista de Educación a Distancia, 4, I-I4.

Kember, D. (2004). Interpreting student workload and the factors which shape students' perceptions of their workload. Studies in Higher Education, 29 (2), I65-I84.

Kember, D., Ng, S., Tse, H., Wong, E. y Pomfret, M. (1996). An examination of the interrelationships between workload, study time, learning approaches and academic outcomes. Studies in Higher Education, 2 I (3), 347-358.

Laborda, C. y Martínez, M. (2006). El seguimiento de la carga de trabajo del estudiantado. La experiencia del tiempo para el trabajo dirigido y autónomo en la experimentación del plan piloto (ECTS) en Pedagogía. En Actas del IV Congreso Internacional de Docencia Universitaria e Innovación (CIDUI 2006). Barcelona, España.

Laborda, C. y Martínez, M. (2007). Mecanismos de seguimiento, coordinación y aseguramiento de la calidad en la transformación de una titulación en base a los criterios del EEES. Educar, 40, I31-159.

Martínez, J. P., Ortega, A., Hernández, A., Salinas, I., García, P., Vicente, L. Martínez, I., y Fernández, J. (2006). Evaluación de la carga discente de la titulación de Ingeniería de Telecomunicación: asignación de créditos ECTS. En Actas del IV Congreso Internacional de Docencia Universitaria e Innovación (CIDUI 2006). Barcelona, España.

Melendro, M., Murga, M. A., Novo, M. y Bautista-Cerro, M. J. (2008). Estrategias formativas innovadoras en Educación Ambiental y para el desarrollo sostenible. RIED: Revista Iberoamericana de Educación a Distancia, II (2), I5-39.

Pogacnik, M., Juznic, P., Kosorok-Drobnic, M., Pogacnik, A., Cestnik, V., Kogovsek, J., Pestevsek, U. y Fernandes, T. (2004). An attempt to estimate students' workload. Journal of Veterinary Medical Education, 3I (3), 255-60.

Plo, F., Sánchez-Velarde, B., Sangüesa, B.y Berrade, L. (2006). Propuesta de diseño curricular de una asignatura de series temporales en el título de grado de estadística. Recuperado el 0I/05/20I5 de < http://www.buenastareas.com/ensayos/Pedagogia/995309.html >.

Real Decreto II25/2003 de 5 de septiembre (BOE de 18/09/2003), por el que se establece el sistema europeo de créditos y el sistema de calificaciones en las 
titulaciones universitarias de carácter oficial y validez en todo el territorio nacional. Boletín Oficial del Estado, 224, 34355-34356.

Rožman, L., Lešer, V. J., Širca, N. T., Dermol, V. y Skrbinjek, V. (20I4). Assessing student workload - tough nut to crack. Paper delivered at the Human Capital without Borders: Knowledge and Learning for Quality of Life, International Conference. 25-27 de Junio de 20I4, Portorož, Slovenia.

Ruiz-Gallardo, J. R., Valdés, A. y Castaño, S. (2006). Prácticum y carga de trabajo. Revista de Investigación Educativa, 24 (2), 557-574.

Ruiz-Gallardo, J. R., Castaño, S., Gómez-Alday, J. J. y Valdés, A. (20I I). Assessing student workload in Problem Based Learning: Relationships among teaching method, student workload and achievement. A case study in Natural Sciences. Teaching and Teacher Education, 27, 619-627.

Sánchez, H. T., Franco, P. y Estrems, M. (2008). Metodología para la estimación de la carga de trabajo del alumno dentro del espacio europeo de educación superior. Experiencias en nuevas tecnologías de innovación docente. En I Jornadas sobre nuevas tendencias en la enseñanza de las ciencias y las ingenierías, 16 a 18 de octubre de 2008. Murcia. Recuperado el I5/0I/20I5 de <http://repositorio.bib.upct.es/dspace/ bitstream/103 I 7// 084/I/mec.pdf $>$.

Stepišnik, J. K., Kolar, O., Širca, N. T. y Lesjak, D. (2007). Student Workload-Student or Teacher Responsibility: Case Study in Higher Education, Slovenia. Paper delivered at the $20^{\text {th }}$ International Congress for Effectiveness and Improvement. Portorož, Slovenia.

Vílchez, J. E. y Escobar, T. (2009). Fundamentos de ciencias físicas y químicas para los nuevos títulos de grado de maestro de primaria. Enseñanza de las Ciencias, Número Extra. VIII Congreso Internacional sobre Investigación en Didáctica de las Ciencias, (pp. 1795-1799). Barcelona: Universidad Autónoma de Barcelona. $<$ http://ensciencias.uab.es/congreso09/numeroextra/art- I 795- I 799.pdf >

Vílchez, J. E. y Escobar, T. (20I4). Uso de laboratorio, huerto escolar y visitas a centros de naturaleza en Primaria: Percepción de los futuros maestros durante sus prácticas docentes. Revista Electrónica de Enseñanza de las Ciencias, 13 (2), 222-24I. Recuperado el 0I/I2/20I 4 de <http://reec.uvigo.es/volumenes/volumen I3/REEC_I3_2_6_ex840.pdf>

Villa, A. y Poblete, M. (Dirs.) (2007). Aprendizaje basado en competencias. Una propuesta para la evaluación de las competencias genéricas. Bilbao: Mensajero/ICE Universidad de Deusto. 
72 - Carga de trabajo autónomo estimada por el estudiante de magisterio en asignaturas de ciencias.

\section{Anexos}

\section{Anexo I}

\begin{tabular}{r|c|c|c|c|c|c}
\cline { 2 - 7 } & \multicolumn{2}{c}{ Td, Tp } & \multicolumn{2}{c}{ To, Tp } & \multicolumn{2}{c}{ Td , To } \\
\hline Tipo de actividades & $\mathbf{p}$ & $\mathbf{t}$ & $\mathbf{p}$ & $\mathbf{t}$ & $\mathbf{p}$ & $\mathbf{t}$ \\
\hline $\begin{array}{r}\text { búsqueda y tratamiento de } \\
\text { Información }\end{array}$ & $0,000 * *$ & $-11,60$ & $0,000 * *$ & $-4,02$ & $0,000 * *$ & $-7,52$ \\
\hline informes de laboratorio & 0,195 & $-1,30$ & 0,178 & 1,35 & $0,000 * *$ & $-6,18$ \\
\hline ejercicios de respuesta abierta & $0,000 * *$ & $-10,15$ & $0,000 * *$ & $-4,35$ & $0,000 * *$ & $-4,84$ \\
\hline ejercicios de respuesta cerrada & 0,672 & 0,42 & $0,004 * *$ & 3,00 & $0,000 * *$ & $-6,27$ \\
\hline resolución de problemas & 0,311 & 3,45 & $0,001 * *$ & 3,45 & $0,000 * *$ & $-6,88$ \\
\hline preparación de exámenes & 0,937 & 0,08 & $0,000 * *$ & 6,39 & $0,000 * *$ & $-10,61$ \\
\hline trabajo con apuntes & $0,022 *$ & 2,30 & $0,000 * *$ & 5,04 & $0,000 * *$ & $-5,57$ \\
\hline
\end{tabular}

Td = Tiempo dedicado; To = Tiempo óptimo; $T p=$ Tiempo planificado $\mathrm{t}$ : $T$ de Student / * p: significación al nivel a $=0,05 /$ ** $\mathrm{p}$ : significación al nivel a $=0,0 \mathrm{I}$

Anexo I. Resultados de la comparación de medias mediante la prueba $T$ para muestras relacionadas. 
Anexo 2

\begin{tabular}{|c|c|c|c|c|}
\hline & \\
\hline & $\mathbf{A}$ & B & C & D \\
\hline Resultados globales & $\begin{array}{l}r=-0,02 \\
p=0,460\end{array}$ & $\begin{array}{l}r=-0,05 \\
p=0,056\end{array}$ & $\begin{array}{l}r=-0,03 \\
p=0,235\end{array}$ & $\begin{array}{c}r= \\
-0,065^{*} * \\
p=0,007\end{array}$ \\
\hline $\begin{array}{l}\text { búsqueda y tratamiento de } \\
\text { Información }\end{array}$ & $\begin{array}{l}r=-0,03 \\
p=0,599\end{array}$ & $\begin{array}{l}r=-0,05 \\
p=0,351\end{array}$ & $\begin{array}{l}r=0,07 \\
p=0,250\end{array}$ & $\begin{array}{l}r=0,01 \\
p=0,812\end{array}$ \\
\hline informes de laboratorio & $\begin{array}{l}r=0,01 \\
p=0,816\end{array}$ & $\begin{array}{l}r=0,06 \\
p=0,369\end{array}$ & $\begin{array}{l}r=0,18^{* *} \\
p=0,006\end{array}$ & $\begin{array}{l}r=0,18 * * \\
p=0,005\end{array}$ \\
\hline ejercicios de respuesta abierta & $\begin{array}{l}r=-0,02 \\
p=0,786\end{array}$ & $\begin{array}{l}r=-0,01 \\
p=0,886\end{array}$ & $\begin{array}{l}r=-0,13 \\
p=0,109\end{array}$ & $\begin{array}{l}r=-0,14 \\
p=0,106\end{array}$ \\
\hline ejercicios de respuesta cerrada & $\begin{array}{l}r=-0,06 \\
p=0,657\end{array}$ & $\begin{array}{l}r=-0,08 \\
p=0,564\end{array}$ & $\begin{array}{l}r=0,03 \\
p=0,846\end{array}$ & $\begin{array}{l}r=-0,11 \\
p=0,436\end{array}$ \\
\hline resolución de problemas & $\begin{array}{l}r=-0,08 \\
p=0,201\end{array}$ & $\begin{array}{l}r=-0,13^{*} \\
p=0,040\end{array}$ & $\begin{array}{l}r=-0,15^{*} \\
p=0,015\end{array}$ & $\begin{array}{l}r=-0,19 * * \\
p=0,004\end{array}$ \\
\hline preparación de exámenes & $\begin{array}{l}r=-0,05 \\
p=0,383\end{array}$ & $\begin{array}{l}r=-0,12 * \\
p=0,031\end{array}$ & $\begin{array}{l}r=-0,04 \\
p=0,511\end{array}$ & $\begin{array}{l}r=-0,14 * * \\
p=0,009\end{array}$ \\
\hline trabajo con apuntes & $\begin{array}{l}r=-0,08 \\
p=0,229\end{array}$ & $\begin{array}{l}r=-0,09 \\
p=0,188\end{array}$ & $\begin{array}{l}r=-0,17^{*} \\
p=0,011\end{array}$ & $\begin{array}{l}r=-0,17^{*} \\
p=0,011\end{array}$ \\
\hline
\end{tabular}

A: Calificación. Actividad vs $\mid$ DifTd,Tp | / B: Calificación. Actividad vs $\mid$ DifTo,Tp

C: Calificación. Actividad vs DifTd,Tp / D: Calificación. Actividad vs DifTo,Tp

r: Coeficiente de correlación de Pearson

* p: significación al nivel a =0,05 / ** p: significación al nivel a =0,0 I

Anexo 2. Coeficientes de correlación de Pearson y significatividad de las pruebas de correlación bivariadas. 